\title{
Estudio experimental y controlado en farmacia comunitaria: influencia de la administración de un
}

\section{complemento alimenticio de ajo en la evolución de la hipercolesterolemia}

\author{
Antucho Espinosa Suances', Antucho Espinosa Estévez², María Espinosa Estévez ${ }^{3}$ \\ 1. Farmacéutico comunitario en A Valenzá, Ourense. Máster en Atención Farmacéutica. 2. Farmacéutico comunitario en A Gudiña, Ourense. Graduado
} en Óptica, Optometria y Audiología. Máster en Biotecnología. 3. Farmacéutica comunitaria en A Gudiña, Ourense.

\section{PALABRAS CLAVE}

Farmacia comunitaria, hipercolesterolemia, colesterol total, complemento alimenticio hipocolesterolemiante de ajo

\section{ABREVIATURAS}

CAH: complemento alimenticio hipocolesterolemiante CT: colesterol total

FC: farmacia comunitaria FEG: farmacia Estévez de A Gudiña.

GC: grupo control.

GI: grupo intervención. MHP: medicamento hipocolesterolemiante de prescripción médica

\section{RESUMEN}

Introducción: Las dislipemias son alteraciones de las lipoproteínas que transportan el colesterol y los triglicéridos, siendo consideradas factor de riesgo de enfermedades cardiovasculares. La eficacia de la utilización de productos vegetales cono el ajo (Allium sativum) para prevenir y tratar dislipemias es discutida. Hay pocos estudios publicados sobre el efecto de estos productos en el ámbito de la farmacia comunitaria.

Objetivos: Estudiar la variación de los valores de colesterol total en pacientes tratados con un complemento alimenticio a base de ajo en cápsulas. Relacionar dichos valores con factores demográficos y con la toma o no de medicamentos hipocolesterolemiantes de prescripción médica (MHPM). Comprobar si los resultados obtenidos concuerdan con los encontrados en la bibliografía.

Material y métodos: Estudio experimental y controlado realizado en una farmacia comunitaria. Los pacientes que aceptaron participar se dividieron aleatoriamente en dos grupos, grupo intervención (GI) que tomaba complemento alimenticio de ajo y grupo control (GC) que no lo tomaba.

Resultados: 59 sujetos, 29 pertenecientes al GC y 30 al GI. Mayor descenso del colesterol total en los que no tomaban MHPM, pero no puede atribuirse al efecto del complemento alimenticio administrado ya que el descenso se produce en los dos grupos. No se encontraron diferencias significativas entre los grupos. En los que ya tomaban MHP, el efecto del complemento alimenticio fue prácticamente inapreciable.

Conclusiones: En las condiciones del estudio realizado, el efecto del complemento alimenticio de ajo no parece ser lo suficientemente efectivo como para reducir los niveles de colesterol de forma significativa.
Recibido: $19 / 1 / 2018$

Aceptado: $27 / 2 / 2018$

Disponible online: $30-3-2018$
Financiación: ninguna.

Conflicto de intereses: ninguno.

Contribución a la autoría: AES participó en el diseño del estudio, el desarrollo de la investigación, el análisis de los resultados, el tratamiento estadistico, la redacción del manuscrito y la revisión del contenido final. AEE en el desarrollo de la investigación, el análisis de los resultados y la redacción del manuscrito. MEE en el desarrollo de la investigación, la redacción del manuscrito y la revisión del contenido final.

Cite este artículo como: Espinosa-Suances A, Espinosa-Estévez A, Espinosa M. Estudio experimental y controlado en farmacia comunitaria: influencia de la administración de un complemento alimenticio de ajo en la evolución de la hipercolesterolemia. Farmacéuticos Comunitarios. 2018 Mar 30; 10(1):33-40. doi:10.5672/FC.2173-9218.(2018/Nol10).001.05 Correspondencia: Antucho Espinosa Suances (antuchoespinosa@redfarma.org)

ISSN 1885-8619 @ SEFAC (Sociedad Española de Farmacia Familiar y Comunitaria). Todos los derechos reservados. 


\section{KEYWORDS}

Community pharmacy, high cholesterol, total cholesterol, garlic-based dietary supplement for lowering cholesterol
Experimental and controlled study on community pharmacy: impact of the administration of a garlic-based dietary supplement on high cholesterol

\section{ABSTRACT}

Introduction: Dyslipidemia is an abnormal level of the lipoproteins that transport cholesterol and triglycerides, and it is considered a risk factor for cardiovascular diseases. The effectiveness of using plant-based products like garlic (Allium sativum) to prevent and treat dyslipidemia is under debate. Few studies have been published on the effect of these products in the context of the community pharmacy.

Objectives: Study the variation of total cholesterol values in patients treated with a garlic-based dietary supplement in capsule form. Link these values to demographic factors and to the use or non-use of prescription cholesterol-lowering drugs. Determine whether the results are consistent with those found in the bibliography.

Materials and Methods: Experimental, controlled study conducted in a community pharmacy. The patients who agreed to participate were divided randomly into two groups, the intervention group (IG), which took a garlic-based dietary supplement, and the control group (CG), which did not take it.

Results: 59 subjects, 29 corresponding to the CG and 30 to the IG. Greater decrease in total cholesterol in the subjects who did not take the prescription cholesterol-lowering drugs, but this result cannot be attributed to the effect of the dietary supplement administered since a decrease occurred in both groups. No significant differences were detected between the groups. In the subjects who were already taking the prescription cholesterol-lowering drugs, the effect of the dietary supplement was practically negligible.

Conclusions: Under the conditions of the study, administration of the garlic-based dietary supplement does not appear to be effective enough to reduce cholesterol levels significantly.

\section{Introducción}

Las enfermedades cardiovasculares (ECV) pueden ser consideradas la gran epidemia del siglo XXI, pues ya son la principal causa de muerte en todo el mundo. Su aumento en términos de incidencia y prevalencia en países en desarrollo tiene implicaciones potencialmente importantes para los países desarrollados [1]. De hecho se prevé que en 2020 serán la principal causa de morbilidad y mortalidad en la mayoría de ellos [2].

La mayor parte de las ECV pueden prevenirse actuando sobre sus factores de riesgo, utilizando estrategias que abarquen a toda la población. Por tanto, en los casos de alto riesgo son fundamentales la detección precoz y el tratamiento temprano, mediante servicios de orientación o la administración de fármacos según corresponda [3].

Las dislipemias son alteraciones del transporte lipídico. Se producen por aumento en la síntesis o retardo en la degradación de las lipoproteínas que transportan el colesterol y los triglicéridos a nivel plasmático. Estas complejas macromoléculas permiten el transporte desde el lugar de absor- ción o síntesis hasta el sitio de utilización [4].

En la actualidad es ampliamente conocida la interrelación entre las alteraciones lipídicas y el riesgo cardiovascular. Son numerosos los factores de riesgo que se asocian a las dislipidemias; por tanto, han de ser tenidos en consideración al iniciar medidas terapéuticas. Además de otras afecciones de indole cardiovascular, diversos tipos de cáncer, el síndrome de ovario poliquístico, hipertensión arterial esencial e incluso enfermedades autoinmunes se han relacionado con los trastornos lipídicos [5].

Desde hace ya varias décadas se vienen utilizando numerosos productos de origen vegetal para prevenir eventos cardiovasculares con mayor o menor éxito. En el caso concreto de las dislipemias, los productos más utilizados son la levadura roja del arroz (Monascus purpureus) [6-8], los policosanoles de la caña de azúcar [9-11], los ácidos grasos omega-3 del pescado azul [12], la lecitina de soja y el ajo. Todos ellos han sido ampliamente utilizados de forma individual, combinados entre sí y también en combinación con fármacos. En cual- quier caso, su aceptación como agentes hipolipemiantes es muy discutida y su eficacia real es actualmente objeto de debate.

Las propiedades beneficiosas del ajo son conocidas desde hace siglos, motivo por el cual se incluye en la lista de alimentos saludables de prácticamente todas las dietas. Hace ya varias décadas, se observó que los efectos positivos del ajo ocurren principalmente a nivel cardiovascular $[13,14]$. Desde entonces se han venido realizando estudios en este sentido y se ha visto que el ajo puede afectar a diferentes parámetros: aumenta la actividad fibrinolítica y el tiempo de coagulación [15], disminuye los niveles de tromboxano B2 y, por tanto, el riesgo trombótico [16], reduce la presión arterial, el colesterol y los triglicéridos $[17,18]$.

Algunos metaanálisis actuales confirman las propiedades del ajo mencionadas anteriormente [19]. Sin embargo, en otros muchos trabajos se concluye que el ajo no presenta dichas propiedades [20-22] y/o si las tuviese, que no son lo suficientemente significativas como para justificar su empleo con tales fines [23]. 
Las conclusiones son contradictorias por diferentes motivos: la falta de estandarización de las dosis de ajo empleadas en cada uno de los estudios, diferencias a la hora de establecer criterios de inclusión/exclusión, diferentes consideraciones en cuanto a estilo de vida y dieta a seguir por los sujetos objeto de estudio, variaciones en los excipientes del preparado de ajo usado en cada caso, diferencias de composición entre los distintos preparados de ajo utilizados [24].

El ajo y los preparados comerciales a base de ajo son relativamente seguros. Sin embargo, se han reportado efectos indeseados, unos más frecuentes que otros. Por orden de frecuencia, los más típicos son la halitosis y el olor corporal debido al uso de ajo crudo; el cual se evita con los preparados a base de ajo libres de olor. Son relativamente frecuentes los efectos a nivel gastrointestinal, como la disfunción y/o dolor gástrico, flatulencias, etc. [25].

Se han descrito casos de problemas más serios, como reacciones alérgicas (incluso anafilaxia), dermatitis, rinitis, angioedema, asma y urticaria. En muy pocos casos, se reportaron alteraciones de la coagulación sanguínea por consumo excesivo o imprudente de preparados de ajo. En cuanto a la relación ajo y coagulación, se han informado 2 casos de interacción del ajo con la warfarina [25]. Sin embargo, Macan et al. demostraron que el uso de ajo comercial era seguro en pacientes a tratamiento con warfarina [26].

Se dispone de pocos estudios publicados sobre el efecto de estos productos en el ámbito de la farmacia comunitaria (FC). Asimismo, se ha constatado que la hipercolesterolemia es una de las patologías de mayor incidencia entre los pacientes de la farmacia Estévez de A Gudiña (Ourense) (FEG), siendo motivo de numerosas consultas relacionadas, tanto con la propia enfermedad, como con los "remedios" utilizados para su tratamiento.

Ante estos hechos, nace la idea de evaluar la efectividad de un complemento alimenticio hipocolesterolemiante (CAH) en un grupo de pacientes de la FEG. Para ello, se utiliza un producto comercializado en España, que contiene $300 \mathrm{mg}$ de bulbo de ajo pulverizado por cápsula. La pauta posológica empleada en este trabajo y recomendada por el laboratorio fabricante es una cápsula en el desayuno $\mathrm{y}$ dos en la cena (1-0-2).

El municipio de A Gudiña está situado en el oriente de la provincia de Ourense, tiene una sola farmacia y cuenta con 1.345 habitantes de acuerdo con el padrón de 2016 [27]. Su población está muy envejecida, más de un 35\% es mayor de 65 años y ha sufrido unos descensos muy importantes en los últimos 10 años.

\section{Objetivos \\ Principal}

Estudiar la variación de los valores de colesterol total (CT) en un grupo de pacientes de la FEG tratados con un CAH durante un período de tiempo.

\section{Secundarios}

Evaluar la variación del CT durante los dos meses y medio del estudio en relación con aspectos socio-culturales y características demográficas de los pacientes (nivel cultural, edad y sexo), así como con la toma concomitante de medicamentos hipocolesterolemiantes de prescripción médica (MHP).

Comprobar si los resultados obtenidos están en concordancia o no con los encontrados en la revisión bibliográfica.

\section{Material y metodos \\ Diseño}

Se trata de un estudio experimental y controlado en el cual se pretende evaluar, tras la administración de un $\mathrm{CAH}$, la variación de los niveles de CT en un grupo de pacientes de la farmacia que padecen hipercolesterolemia.

\section{Pacientes}

Los sujetos objeto de estudio fueron todos los pacientes que acudieron a la FEG en el periodo de tiempo comprendido entre el 1/12/2016 y el $15 / 3 / 2017$, que aceptaron libremente participar en el estudio y que cumplian los criterios de inclusión/exclusión. Se dividieron en dos grupos, grupo control (GC) y grupo intervención (GI), asignados aleatoriamente por orden de aceptación en el estudio, correspondiendo los números pares al GC y los impares al GI.
Criterios de inclusión

- Ser mayor de 18 años.

- Tener el CT >200 mg/dL.

- Querer y poder participar en las 3 sesiones del estudio.

- Aceptar la administración diaria de CAH durante los 4 meses de duración del estudio.

\section{Criterios de exclusión}

- Padecer alguna incapacidad física o psíquica que le impida al sujeto participar en el estudio.

- Haber iniciado algún tratamiento farmacológico o con $\mathrm{CAH}$ en los últimos 3 meses.

- Ser mujer y estar embarazada o lactando.

- Padecer algún trastorno de coagulación sanguínea.

- Padecer hipotensión arterial.

- Ser alérgico al ajo o a algún componente del CAH.

\section{Variables cualitativas}

- Sexo: hombre/mujer.

- Nivel cultural: primaria, secundaria, bachillerato/formación profesional, estudios universitarios.

- Toma del CAH: sí/no. Toma de MHP: sí/no.

\section{Variables cuantitativas}

- Edad.

- CT.

Registro de datos e instrumentos de medida

Los datos personales y los valores de las variables estudiadas, correspondientes a cada uno de los sujetos del estudio, figuran en la Hoja de recogida de datos (figura 1). Posteriormente se volcaron a una hoja de MS Excel ${ }^{\circledR}$ para poder ser tratados y analizados mediante un software estadístico apropiado.

La determinación del CT se realizó por los farmacéuticos adjuntos de la FEG. El aparato empleado fue el fotómetro de reflexión automática Reflotrón ${ }^{\circledR}$ Plus, número de serie 5069397 [28].

Se utilizó una Hoja de consejos para prevenir los niveles altos de colesterol diseñada por la Sociedad Española de Farmacia Familiar y Comunitaria (SEFAC) [29], que recoge las recomendaciones generales de tipo saludable que han de seguir los sujetos del estudio. 
(1) Paciente $n^{\circ}$

(2) Nombre y apellidos

(3) Dirección

(4) Teléfono

(5) Correo-e

(6) Edad

(7) Grupo (márquese con X):

Control $\square \quad$ Intervención $\square$

(8) Sexo (márquese con $X$ ):

$\mathrm{HD} \quad \mathrm{M} \square$

(9) Nivel cultural (márquese con X):
$\square$ E. Primaria
$\square$ ESO
$\square$ Bachillerato/FP
$\square$ Universidad

(10) Padece el paciente alguna incapacidad física o psíquica, que le impida participar en el estudio (márquese con X):

Si $\square \quad$ No $\square$

(11) Mujer embarazada o lactando (márquese con X):

Si $\square \quad$ No $\square$

(12) Padece algún trastorno de la coagulación sanguínea (márquese con X):

Si $\square \quad$ No $\square$

(13) Padece hipotensión arterial (márquese con X):

Sí $\square \quad$ No $\square$

(14) Es alérgico al ajo o algún componente del CAH (márquese con X):

Si $\square \quad$ No $\square$

(15) Ha iniciado algún tratamiento farmacológico o con CAH en los últimos 3 meses (márquese con X):

Si $\square \quad$ No $\square$

(16) Toma algún medicamento de prescripción médica para disminuir el nivel de colesterol (márquese con X):

Si $\square \quad$ No $\square$

(17) Datos analíticos:

\begin{tabular}{|l|l|l|l|}
\hline Sesión & 1a Visita & 2a Visita & 3a Visita \\
\hline $\mathbf{C T}(\mathbf{m g} / \mathbf{d L})$ & & & \\
\hline
\end{tabular}

Figura 1 Hoja de recogida de datos 


\section{Procedimiento}

El reclutamiento de los sujetos del estudio se llevó a cabo en el mostrador de la propia farmacia, ofreciéndoles participar en el estudio. Los que aceptaron y cumplieron los criterios de inclusión/exclusión se les cumplimentó la hoja de recogida de datos, se les asignó el grupo al que van a pertenecer (GC o GI), se les explicó de forma pormenorizada (verbal y escrita) el contenido del estudio y se les dio una cita para la primera sesión.

En la primera sesión se explicó más detalladamente en qué consistía el estudio y firmaron el consentimiento informado para participar en él. Se les determinó el CT y se cumplimentó la hoja de recogida de datos con los valores encontrados. Se le entregó a cada miembro del GC una hoja de consejos para prevenir los niveles altos de colesterol y a cada miembro del GI, además, un envase del CAH. Por último, se le recordó a cada sujeto la importancia de cumplir escrupulosamente lo acordado y el no abandonar su MHP.

En la segunda sesión, al cabo de 8 semanas, se siguió la misma operatoria que en la $1^{\text {a }}$, partiendo de la determinación del CT.

En la tercera sesión, al cabo de otras 8 semanas, se determinó el CT y se cumplimentó la hoja de recogida de datos con los nuevos valores.

La adherencia al tratamiento se comprobó en el momento de la recogida de los envases del CAH en la farmacia, mediante la comprobación de que efectivamente los envases anteriormente suministrados estuviesen ya vacíos.

El método utilizado para la selección de los sujetos fue un muestreo no probabilístico, ya que se le ofreció a todos los pacientes de la farmacia con hipercolesterolemia la posibilidad de participar o no en el estudio; de ahí las limitaciones obvias de las conclusiones a las que se pueda llegar. $\mathrm{Al}$ incluir a toda la población accesible no se realizó el cálculo del tamaño muestral.

Se aplicaron técnicas estadísticas para contrastar si había diferencias significativas en las determinaciones de CT a medida que se administraba el CAH: Contrastes de Hipótesis para Muestras Independientes y Pruebas para Medidas Repetidas.

Se han añadido unas estimaciones no paramétricas de la función de
Tabla 1 Medicamentos hipolimemiantes de prescripción médica que toman los sujetos del estudio

\begin{tabular}{|l|c|c|}
\hline Medicamento y dosis & $\begin{array}{c}\text { Grupo } \\
\text { control }\end{array}$ & $\begin{array}{c}\text { Grupo } \\
\text { intervencion }\end{array}$ \\
\hline Atorvastatina $10 \mathrm{mg}$ & 3 & 2 \\
Atorvastatina $20 \mathrm{mg}$ & 4 & -- \\
Atorvastatina $40 \mathrm{mg}$ & -- & 1 \\
Simvastatina $10 \mathrm{mg}$ & 2 & -- \\
Simvastatina $20 \mathrm{mg}$ & 2 & 4 \\
Ezetimiba $10 \mathrm{mg} /$ Atorvastatina $20 \mathrm{mg}$ & 1 & -- \\
Pravastatina $40 \mathrm{mg} /$ Fenofibrato $160 \mathrm{mg}$ & 1 & 2 \\
Rosuvastatina $5 \mathrm{mg}$ & 1 & -- \\
Rosuvastatina $10 \mathrm{mg}$ & -- & 3 \\
Pitavastatina $2 \mathrm{mg}$ & -- & 2 \\
\hline
\end{tabular}

regresión: Estimaciones tipo Kernel, que no asumen ningún modelo específico de la función de regresión y que explicaría el nivel de colesterol para cada medida en función de la edad.

\section{Resultados y discusión}

Se ofreció participar en el estudio a un total de 96 pacientes, de los cuales 5 rechazaron la oferta, 13 cumplieron los criterios de exclusión y 15 incumplieron los criterios de inclusión. A lo largo del estudio se produjeron 4 abandonos, en la segunda y tercera visita por orden médica $u$ otras circunstancias ajenas al motivo por el cual se realizó el estudio. Por lo tanto, el análisis final fue llevado a cabo sobre los resultados de un total de 59 sujetos, 29 del GC y 30 del GI.

Veinticinco $(42,4 \%)$ sujetos del estudio son hombres, de los cuales 14
$(56,0 \%)$ toman MHP. En el caso de las mujeres lo toman $16(47,1 \%)$ (tabla 1$)$. El 79,7 \% de los individuos tiene un nivel cultural menor o igual a la ESO. La edad, debido al grado de envejecimiento de la población, tiene un valor medio en torno a los 59,0 años ( $D E=11,1$; Min=28 y Max=83).

En la tabla 2 se presenta la media de las tres determinaciones de CT teniendo en cuenta el GC y el GI y en función de si tomaban algún MHP o no. Existe una elevada dispersión $(\mathrm{DE}=43,5)$ en las medidas de la primera visita del GC que no toma MHP, lo cual contrasta con una $\mathrm{DE}=15,0$ unidades del GI, que sí toma MHP.

Se puede observar un descenso medio más notable en los sujetos que no toman MHP. Puesto que este descenso se da tanto para el grupo control como para el de intervención, no puede atribuirse al efecto del CAH administrado.
Tabla 2 Nivel medio, máximo, mínimo y DE de colesterol total para cada medida realizada en el estudio en función de la pertenencia a los grupos control e intervención y de la toma o no de medicamentos hipocolesterolemiantes de prescripción médica

\begin{tabular}{|c|c|c|c|c|c|c|}
\hline \multirow{2}{*}{$\begin{array}{l}\text { Toma } \\
\text { MHP }\end{array}$} & \multirow[b]{2}{*}{ Grupo } & & \multicolumn{3}{|c|}{ Colesterol total $\mathrm{mg} / \mathrm{dL}$} & \multirow{2}{*}{$\begin{array}{c}\text { p-Valor } \\
\text { C-I }\end{array}$} \\
\hline & & & m (DE) & Máximo & Mínimo & \\
\hline \multirow{6}{*}{ NO } & \multirow{3}{*}{$\mathrm{GC}$} & 1 a visita & $231,6(43,5)$ & 365,0 & 200,0 & \multirow{6}{*}{$\begin{array}{l}0,982 \\
0,847 \\
0,931\end{array}$} \\
\hline & & $2^{\text {a }}$ visita & $226,8(26,7)$ & 297,0 & 180,0 & \\
\hline & & $3^{\text {a }}$ visita & $215,6(16,9)$ & 240,0 & 189,0 & \\
\hline & \multirow{3}{*}{$\mathrm{Gl}$} & $1^{\text {a }}$ visita & $231,9(24,7)$ & 308,0 & 207,0 & \\
\hline & & $2^{\text {a }}$ visita & $229,1(36,0)$ & 295,0 & 175,0 & \\
\hline & & $3^{\text {a }}$ visita & $216,4(31,5)$ & 268,0 & 152,0 & \\
\hline \multirow{6}{*}{ sí } & \multirow{3}{*}{$\mathrm{GC}$} & $1^{\text {a }}$ visita & $206,8(19,8)$ & 255,0 & 180,0 & \\
\hline & & $2^{\text {a }}$ visita & $197,1(20,8)$ & 247,0 & 165,0 & \\
\hline & & $3^{\text {a }}$ visita & $203,4(37,1)$ & 290,0 & 163,0 & \\
\hline & \multirow{3}{*}{$\mathrm{Gl}$} & $1^{\text {a }}$ visita & $224,3(15,0)$ & 249,0 & 203,0 & 0,014 \\
\hline & & $2^{\text {a }}$ visita & $224,8(27,0)$ & 282,0 & 181,0 & 0,004 \\
\hline & & $3^{\text {a }}$ visita & $228,9(39,4)$ & 294,0 & 165,0 & 0,084 \\
\hline
\end{tabular}

En la última columna figura el p-valor de la prueba t-de Student para muestras independientes comparando los valores medios de cada visita entre los grupos control e intervención 


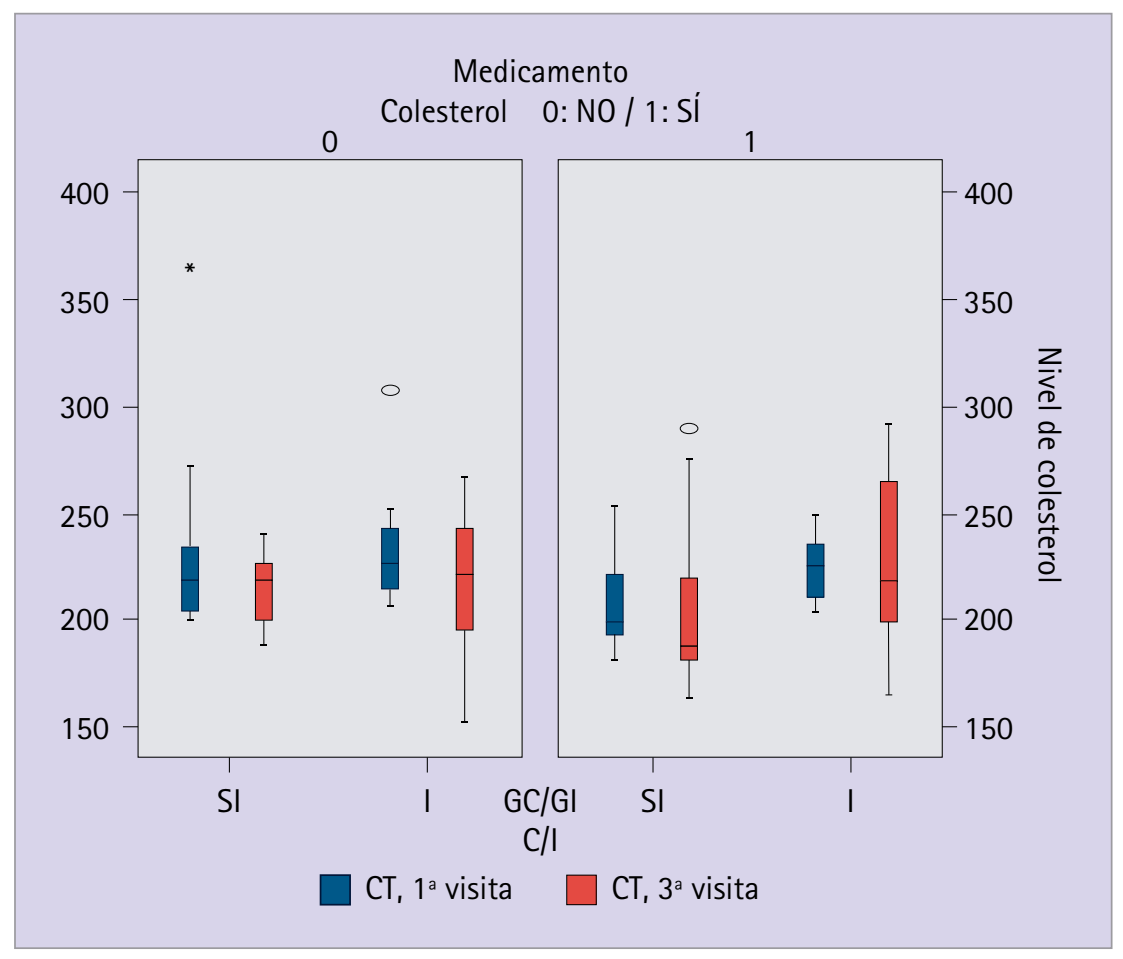

Figura 2 Diagramas de cajas del nivel de CT en función de los GC/GI y de la administración o no de MHP. Comparativa entre la primera y la tercera visita

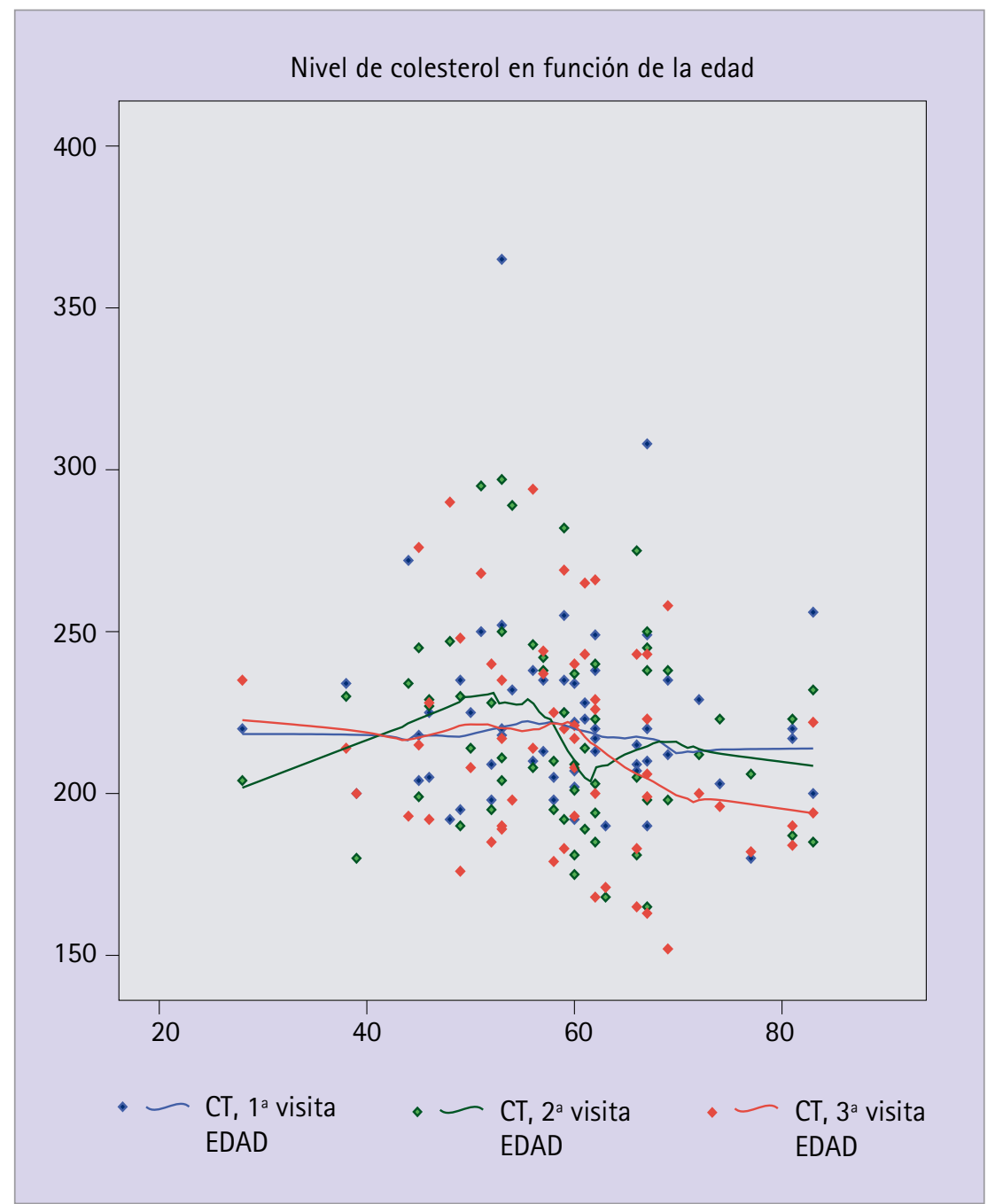

Figura 3 Diagrama de dispersión del nivel de CT en función de la edad para cada medida. Las líneas representan una suavización no paramétrica de cada conjunto de puntos
En la figura 2 se muestran los diagramas de cajas correspondientes a la primera y tercera medida. Dada la gran dispersión de los datos es difícil observar diferencias significativas entre los grupos en los cuales se ha administrado el MHP o no.

En la figura 3 está representado un diagrama de dispersión del nivel de CT en función de la edad del paciente. Como puede verse, la dispersión hace difícil una interpretación clara del efecto del componente objeto de estudio. La variabilidad es tan grande, que es difícil observar una tendencia clara, pero los resultados no presentan diferencia alguna.

Análisis estadístico: comparación de medias

Los resultados no fueron significativos, no pudiendo rechazar la hipótesis nula de igualdad de distribuciones dado que los p-valores eran superiores al 10\% en todos los casos.

Una vez comprobada la normalidad de las medidas de CT utilizando el Contraste de Kolmogorov-Smirnov, se aplicaron test de comparación de medias para ver si las diferencias eran significativas entre los grupos control e intervención. Dado que los resultados pueden verse influidos por el hecho de tomar MHP, se aplicaron estas técnicas para cada grupo por separado.

Los resultados de los p-valores se adjuntan en la última columna de la tabla 3. Sólo son significativos los contrastes correspondientes a los individuos que toman MHP. Los resultados muestran que las diferencias entre las medias de CT para la primera y segunda medida entre los grupos control e intervención son significativas; sin embargo, puede afirmarse con un $5,0 \%$ que la media del grupo de intervención es superior a la media del grupo control para esas dos medidas.

También se aplicaron técnicas específicas para medidas repetidas teniendo en cuenta el factor GC/GI pero los resultados no fueron significativos.

Análisis de medidas repetidas

Se ha realizado el análisis considerando el nivel de colesterol como medida repetida en tres instantes distintos, teniendo en cuenta el factor inter-sujeto de GC/GI.

Los individuos que no toman MHP sí presentan diferencias significativas 
entre las tres medidas de colesterol, pero estas no son debidas al efecto del CAH, véase valores de la tabla 3, representando los p-valores de los contrastes realizados. Quizás puedan ser debidas a una mejora en la alimentación o a otros hábitos saludables.

Por otra parte, parece lógico que en los individuos que ya estaban a tratamiento con algún MHP, el efecto del nuevo complemento alimenticio sea casi inapreciable.

El objetivo principal de este trabajo es estudiar la variación de los valores de CT en pacientes de la FEG tratados con un CAH durante un período de tiempo. Tal y como reflejan los resultados obtenidos, hemos de decir que el CAH empleado no logra reducir los niveles de CT de forma significativa. Con los datos de las tres determinaciones, no se observa una diferencia tal entre los valores del GC en comparación con el GI como para considerar que el CAH tiene eficacia como agente hipolipemiante. La fragmentación de la muestra, ya de por sí reducida, en cuatro grupos, dificulta aún más la obtención de significación estadística en los resultados.

Por otra parte, se observa un descenso medio más notable en los sujetos que no tomaban MHP. Podría pensarse que el efecto del $\mathrm{CAH}$ es superior en sujetos sin tratamiento farmacológico previo en comparación con los individuos con tratamiento. Sin embargo, este razonamiento no es válido en este caso, puesto que no existen diferencias entre GC y GI; es decir, tratados y no tratados con el CAH presentan la misma tendencia de variación en los niveles de CT.

El aspecto más destacable de este estudio quizás sea el hecho de no encontrar diferencias en los niveles de CT al hacer la comparativa entre las distintas variables a estudio: GC/GI, niveles de CT por rangos de edad y medicados/ no medicados con fármacos hipolipemiantes de prescripción médica.

Tal y como se ha explicado en esta introducción, algunos estudios defienden que el ajo presenta numerosas propiedades beneficiosas para la salud y en concreto para prevenir y tratar las dislipemias [13-19]. Sin embargo, los resultados de estos estudios están más en concordancia con los obtenidos por otros autores, que no atribuyen al ajo poder suficiente como para ser empleado en dislipemias de manera individual [20-23].

Tabla 3 p-valores de las pruebas de análisis de medidas repetidas en función de la toma o no de MHPy de los GC/GI

\begin{tabular}{|c|c|c|c|}
\hline \multirow{2}{*}{\multicolumn{2}{|c|}{ Pruebas medidas repetidas }} & \multicolumn{2}{|c|}{ MHP } \\
\hline & & NO & sí \\
\hline \multirow{4}{*}{ Medidas } & Esfericidad asumida & 0,041 & 0,647 \\
\hline & Greenhouse-Geisser & 0,046 & 0,601 \\
\hline & Huynh-Feldt & 0,041 & 0,618 \\
\hline & Limite-inferior & 0,076 & 0,514 \\
\hline \multirow{4}{*}{ Medidas * GC/GI } & Esfericidad asumida & 0,987 & 0,677 \\
\hline & Greenhouse-Geisser & 0,981 & 0,628 \\
\hline & Huynh-Feldt & 0,986 & 0,645 \\
\hline & Limite-inferior & 0,909 & 0,536 \\
\hline
\end{tabular}

De hecho, en dichos metaanálisis se obtienen reducciones de CT del 9-12\% con respecto a placebo (mayores que en el presente estudio) y aun así consideran que el poder hipolipemiante de los preparados de ajo debe ser testado en revisiones más extensas.

\section{Limitaciones del estudio}

Sin duda, la dificultad para mantener un estilo de vida saludable por parte de los individuos en sus respectivos domicilios ha sido la principal limitación. Muchas de ellas son personas en situaciones especiales (edad avanzada, situación laboral complicada, cambios de dieta, cambios en el tratamiento farmacológico habitual, etc.), lo cual dificulta la constancia y las pautas a seguir marcadas en el estudio. Aunque también es cierto que estas son las condiciones reales en las que los pacientes ambulatorios desarrollan su vida.

Otra limitación a tener en cuenta es la no disponibilidad en la FEG de un aparato capaz de desglosar los niveles de CT de las mediciones en CHDL (High Density Lipoprotein: HDL) y CLDL (Low Density Lipoprotein: LDL). De esta forma, sería posible evaluar de qué manera está actuando el CAH: reduciendo el CHDL, el CLDL, ninguno de ellos o ambos.

\section{Utilidad práctica del estudio}

Como ya se ha dicho en la introducción, los estudios realizados en FC en relación con el ajo y sus propiedades hipolipemiantes son muy escasos o inexistentes. El presente trabajo ha servido para evaluar los efectos del ajo a nivel farmacéutico-paciente, con los múltiples sesgos que ello acarrea y lejos de las condiciones y características de un estudio más amplio. A pesar de que los resultados obtenidos parecen indicar que el complemento de ajo no tiene un gran poder hipolipemiante, queda abierta una puerta a trabajos posteriores, con muestras más amplias, que subsanen las limitaciones descritas y permitan dotar de significación estadística a los resultados que se obtengan.

\section{Conclusiones}

Evaluación de los niveles de CT

- Los valores de CT del conjunto de sujetos participantes en el estudio, tanto del GC como del GI, no varían de forma clara ni presentan algún tipo de tendencia de variación.

- No se encontraron diferencias significativas al comparar los niveles de CT de los sujetos pertenecientes al GC y GI entre sí.

- Se concluye, con la limitación del reducido tamaño de la muestra, que el efecto del CAH administrado no es lo suficientemente potente como para reducir los niveles de CT de forma significativa.

Evaluación de las variables a estudio

- Las características socio-culturales y demográficas (edad, sexo y nivel cultural) de los pacientes no influyen en los niveles de CT de los sujetos ni en la evolución de dichos niveles durante el estudio.

- No existen diferencias significativas al comparar los niveles de CT de los sujetos que no tomaban MHP con respecto a los que sí tenían algún tipo de tratamiento farmacológico hipolipemiante, al administrarles simultáneamente el CAH. 
Se concluye que el hecho de estar o no estar a tratamiento con algún MHP no influye significativamente en el efecto del CAH sobre el CT.

\section{Agradecimientos}

A Nicanor Floro Andrés Rodríguez, farmacéutico comunitario, $\mathrm{y}$ a Ana Pérez González, profesora de Estadística de la Universidad de Vigo, por su inestimable ayuda en la realización de este trabajo.

\section{Referencias bibliográficas}

1. Celermajer DS, Chow CK, Marijon E, Anstey NM, Woo KS. Cardiovascular disease in the developing world prevalence's, patterns, and the potential of early disease detection. Am Coll Cardiol. 2012; 60(14):1207-1216. https:// doi.org/10.1016/j.jacc.2012.03.074

2. Gersh BJ, Sliwa K, Mayosi BM, Yusuf S. Novel therapeutic concepts: the epidemic of cardiovascular disease in the developing world: global implications. European Heart Journal. 2010; 31(6):642-648. https://doi. org/10.1093/eurheartj/ehq030

3. Organización Mundial de la Salud. Enfermedades cardiovasculares. [Monografía enInternet]. Nota descriptiva. Enero 2015. [Consultada 18/3/2016]. Disponible en: http://www.who.int/ mediacentre/factsheets/fs317/es/

4. Fakhrzadeh H, Tabatabaei-Malazy 0. Dyslipemia and Cardiovascular Disease. Dislipemia from prevention to treatment. Croatia: Ed. Roya Kelishadi. 2012; ISBN: 978-953-307-904-2.

5. Chiqui RA, Bermúdez V, Añez R, Salazar J, Rojas J, Landi A. Prevalencia de dislipidemia y factores asociados en la ciudad de Cuenca, Ecuador. Síndrome Cardiometabólico. 2014; 4(2):31-41.

6. Marín F, Martín M, Sacristán A, Ocaña JC, Llopis B, Fernández V et al. Efecto reductor del colesterol de una combinación de levadura roja de arroz y policosanol. Revista de Fitoterapia. 2016; 16(1):5-14.

7. Lin J, Zhang J, Shi Y, Grimsgaard S, Alraaek T, Fonnebo V. Chinese red yeast rice [Monascus Purpureus) for primary hyperlipidemia: A meta-analysis of randomized controlled trials. Chinese Medicine. 2006; 1(4). doi: 10.1186/1749-8546-1-4. https:// doi.org/10.1186/1749-8546-1-4

8. Li Y, Jiang L, Jia Z, Xin W, Yang S, Yang $Q$ et al. Metaanalysis of red yeast rice: efective and relatively safe alternative approach for dyslipidemia. PLoS One. 2014; 9(6): e98611. https://doi.org/10.1371/journal. pone.0098611
9. Varady KA, Wang Y, Jones PJ. Role of policosanols in the prevention and treatment of cardiovascular disease. Nutr Rev. 2003; 61(11):376-383. https://doi.org/10.1301/nr.2003. nov.376-383

10. Gouni-Berthold I, Berthold HK. Policosanol: clinical pharmacology and therapeutic significance of a new lipid-lowering agent. Am Heart J. 2002; 143(2):356-365. https://doi. org/10.1067/mhj.2002.119997

11. Menéndez R, Amor AM, Rodeiro I, González RM, González PC, Alfonso JL et al. Policosanol modulates HMGCoA reductase activity in cultured fibroblasts. Arch Med Res. 2001; 32(1):8-12. https://doi.org/10.1016/ S0188-4409(00)00265-4

12. Chan D, Pang J, Barrett H, Sullivan DR, Burnett J, Van Bockxmeer F et al. Omega-3 Fatty Acid Ethyl Esters Diminish Postprandial Lipemia in Familial Hypercholesterolemia. J Clin Endocrinol Metab. 2016; 101(10):3732-3739. https://doi.org/10.1210/jc.2016-2217

13. Mader FH. Treatment of hyperlipidaemia with garlic-powder tablets. Evidence from the German association of general practitioners' multicentric placebo-controlled double-blind study. Arzneimittel-Forschung. 1990; 40(10):1111-1116.

14. Bakhsh R, Chughtai MI. Influence of garlic on serum cholesterol, serum triglycerides, serum total lipids and serum glucose in human subjects. Die Nahrung. 1984; 28(2):159-163. https:// doi.org/10.1002/food.19840280213

15. Gadkari JV, Joshi VD. Effect of ingestion of raw garlic on serum cholesterol level, clotting time and fibrinolytic activity in normal subjects. Journal of Postgraduate Medicine. 1991; 37(3):128-131.

16. Ali M, Thomson M. Consumption of a garlic clove a day could be beneficial in preventing thrombosis. Prostaglandins Leukotrienes and Essential Fatty Acids. 1995; 53(3):211212. https://doi.org/10.1016/09523278(95)90118-3

17. Steiner M, Khan AH, Holbert D, Lin RI. A double-blind crossover study in moderately hypercholesterolemic men that compared the effect of aged garlic extract and placebo administration on blood lipids. Am J Clin Nutr. 1996; 64(6):866-870. https:// doi.org/10.1093/ajcn/64.6.866

18. Mahmoodi M, Islami MR, Karam GRA, Khaksari M, Sahebghadam Lotfi A, Hajizadeh MR et al. Study of the effects of raw garlic consumption on the level of lipids and other blood biochemical factors in hyperlipidemic individuals. Pak J Pharm Sci. 2006; 19(4):295-298.

19. Varshney R, Budoff MJ. Garlic and Heart Disease. J Nutr.
2016; 146(2):416-421. https://doi. org/10.3945/jn.114.202333

20. Luley C, Lehmann-Leo W, Möller B, Martin T, Schwartzkopff W. Lack of efficacy of dried garlic in patients with hyperlipoproteinemia. Arzneimittel-Forschung. 1986; 36(4):766-768.

21. Khoo YS, Aziz Z. Garlic supplementation and serum cholesterol: a meta-analysis. J Clin Pharm Ther. 2009; 34(2):133-145. https://doi.or$\mathrm{g} / 10.1111 / \mathrm{j} .1365-2710.2008 .00998 . \mathrm{x}$

22. Gardner CD, Lawson LD, Block E, Chatterjee LM, Kiazand A, Balise RR et al. Effect of Raw Garlic vs Commercial Garlic Supplements on Plasma Lipid Concentrations in Adults With Moderate Hypercholesterolemia: A Randomized Clinical Trial. Arch Intern Med. 2007; 167(4):346-353. https://doi. org/10.1001/archinte.167.4.346

23. Stevinson C, Pittler MH, Ernst E. Garlic for treating hypercholesterolemia. A meta-analysis of randomized clinical trials. Ann Intern Med. 2000; 133(6):420-429. https://doi.org/10.7326/0003-4819133-6-200009190-00009

24. Qidwai W, Ashfaq T. Role of Garlic Usage in Cardiovascular Disease Prevention: An Evidence-Based Approach. Evid Based Complement Alternat Med. 2013; 2013:125649. doi: 10. 1155/2013/125649.

25. Borrelli F, Capasso R, Izzo AA. Garlic (Allium sativum L.): adverse effects and drug interactions in humans. Mol Nutr Food Res. 2007; 51(11):13861397. https://doi.org/10.1002/mnfr. 200700072

26. Macan H, Uykimpang R, Alconcel M, Takasu J, Razon R, Amasage $\mathrm{H}$ et al. Aged garlic extract may be safe for patients on warfarin therapy. J Nutr. 2006; 136(Supl.3):793-795. https:// doi.org/10.1093/jn/136.3.793S

27. Instituto Nacional de Estadística. Cifras oficiales de población resultantes de la revisión del Padrón municipal. Ourense: Población por municipios y sexo. [Internet]. [Consultada: 08-112017]. Disponible en: http://www.ine. es/jaxiT3/Tabla.htm?t=2885ct $=0$

28. Roche [Internet] . Productos de diagnóstico: Reflotron ${ }^{\circledR}$ Plus. [Consultada: 08-11-2017]. Disponible en: http:// www.roche.es/Diagnostics/Productos/ point_of_care/atencionprimaria_farmacia_y_paciente/determinaciones_ bioquimicas_rapidas/reflotron_plus. html

29. Consejos para prevenir los niveles altos de colesterol. Recomendaciones de la Sociedad Española de Farmacia Familiar y Comunitaria a la población. [Internet]. [Consultada: 08-11-2017]. Disponible en: https://www.sefac.org/ sites/default/files/sefac2010/private/ documentos_sefac/documentos/SEF_ FR_Colesterol_V.pdf 